\title{
Predicting the Economic Impact of Using Renewable Energy by Modelling Through Artificial Intelligence Techniques
}

\author{
Mioara Chirita ${ }^{1}$, Daniela Ancuta Sarpe ${ }^{2}$, Nicoleta Cristache ${ }^{3}$, Adrian $\mathrm{Micu}^{4}$, \\ Alexandru Capatina ${ }^{5}$
}

\begin{abstract}
Diversification of energy supplies is one of the main priorities of the energy policy of the developed countries. The major objective of this research is the model for predicting the economic impact of renewable energy using artificial intelligence techniques. This has been achieved by using the neural networks for the various issues related to renewable energy.

The designed model consist in identification of those macroeconomic indicators that are required for the database creation, validation and testing in the view of obtaining the smallest error in the validation set for predicting the renewable energy impact upon the economy. The performance of the model was also revealed by comparing control graphs.
\end{abstract}

Key words: renewable energy, economic growth, forecast, macroeconomic indicators

\section{Introduction}

Taking into account the limitations of the estimated fossil fuels, the necessity of identifying and exploiting other alternative energy supplies emerges, such as the renewable energy sources that contribute to the ecological balance of the planet.

The renewable sources of energy (wind, solar, hydropower energy, hydroelectric energy, geothermal energy, biomass and biofuels) are alternative sources to the fossil fuels that contribute to the reduction of greenhouse gas emissions, to the diversification of energy supplies and to the reduction of dependence on the volatile and unreliable markets of fossil fuels, especially oil and gas.

The limited resources of the planet must be used wisely in a more sustainable way. Our society relies on metals, minerals, fuels, water, wood, fertile soil and fresh air, which constitute vital factors for maintaining the proper running of our economy. If all these limited sources are consumed faster than they can regenerate, then significant unbalances can occur. Europe relies on the rest of the world for resources, such as fuels and raw material, which are embedded in products imported from outside EU. The deficits and price volatility of commodities could cause instability in some regions of the world; consequently the increase of efficient use of resources becomes essential (Calvert and Simandan, 2010). 
An interesting research conducted in Turkey outlines that the remarkable renewable energy potential of this country and suggest the renewable sources as a solution for Turkey's energy-related challenges (Çetin and Vardar, 2014).

A viable solution could be the replacement of the main energy sources with alternatives ones, which provide higher efficiency and reduce the environmental impact during their life cycle. In order to meet the demand for energy resources covered by renewable supplies, combined with classical ones, the European states have developed various energy strategies, focussing mainly on increasing the economic efficiency, the use of primary energy resources and of the converted ones (Khaligh and Onar, 2009).

European Union is highly involved in the global fight against climate change, by setting some of the world's most ambitious climate as well as strategic energy targets and integrating of more and more renewable energy (Ya, 2016).

The topic of the present research aims to provide answers to some current issues of utmost importance, regarding the economy of numerous states especially today, when the increase of energy security is a priority by ensuring the supply of energy resources and by reducing the dependence on imported energy (Global, 2015). Being a complex reality, the present study focussed on the evolution, discovery and promotion of energy resource supplies, mainly due to an important aspect related the global supplies of oil that can sustain the current level of consumption only by 2014 and that of natural gas up to 2070, whereas the world supplies of coal could sustain the consumption for a period of more 200 years, even if the rate of consumption increases (Sims et al., 2007; Speirs et al., 2015).

Many forecasts indicate an economic growth, which would imply a significant increase in energy consumption. (Hymans, 2016; Yáñez-Arancibia et al., 2014)

Several experimental models have been achieved so far in this regard that use artificial intelligence and neural networks, which employed various economic indicators as input parameters, out of which a model has been selected whose predicted data converged best with the data reported officially by the internationally-recognized bodies. (Alam et al., 2006; Cao, 2006). The model creates an artificial neural network, tested by input-output data, obtained through economic and statistical methods.

In order to meet the requirements of the predictability characteristic, the influence of past values upon the indicator's present and future values was considered. The model was developed, trained and tested using parameters for different periods of time.

\section{Theoretical Framework}

Artificial intelligence was chosen for this prediction model as it was based on historical data of a system and consequently, better understood and appreciated than other theoretical and empirical methods. Artificial intelligence can be used in this field because it offers innovative design-problem solving and provides almost an instant expertise on the effect of a proposed change in a model. The approach that uses these methods is the best option when the information is available and when fast and accurate enough algorithms cannot be obtained for processing. (Kornienko, 2015).

The main reason for this choice is that modern research starts from statistical hypotheses on the observed phenomenon, its deduced logical consequences and then comparing the 
information with the available data. If they are consistent, then the hypotheses are justified, or at least until a new, more accurate analysis is made.

It is a well-known and also demonstrated fact that artificial neural networks have been developed to solve various problems as they can develop a memory able to make associations and connections between different input sets corresponding to certain output sets.

The main advantage of using artificial neural networks lies in their flexibility to distortions in input data and their ability to acquire, providing ideal solutions for a wide range of classifying and signal recognition problems as well as in forecasting and system modelling, especially where the process is characterized by a high complexity (Sözen et al., 2005).

In order to achieve a good predictive network performance, it is essential to train it with data that are significant for the actual process. Relevant research literature shows that training a network based on data taken randomly and that covers the entire operating field, provides the best representation and generalization capacity. (Kalogirou, 2001)

Russell and others stated that for an empirical model that is used in a closed-loop control system, the data acquisition training must be carried out under the same circumstances. (Russell and Norvig, 2003)

The optimum number of neurons in the hidden layer, the number of training epochs or the mean squared error value cannot be predicted accurately before a proper evaluation of the network performance in several sets of experiments. The optimum number of those values can be determined only experimentally and depends on the type of problem and the structure of the neural network (Mellit, 2003), Grenander 1981), Geman, 1982, Feng et al. 2015).

Another issue that can arise is related to the resources and data acquisition used in the experiment (Parker, 1982), Rumelhart, et al. 1986).

All the above-mentioned aspects are highly important and in this regard, we chose to compare the data form different sources in order to identify their accuracy and thus to avoid the possibility of introducing false data.

\subsection{The importance of model building}

Based on certain scientific papers, on statistical and economic data and on information systems, the present research focused on renewable energy sources, highlighting the necessity of developing a model to predict the impact of renewable energy upon the economy (Zhang et.al. 2013).

The impact of developing renewable energy requires imperatively models to predict, some logic programs that would allow decision-making and data analysis by selecting alternatives. In the view of building these models, in an early stage it was necessary to identify the economic indicators that have a major impact upon the development of renewable energies in general.

Thus, the present study aimed at comparing, analysing and building a model using artificial intelligence and certain economic indicators that are important in economic prediction regarding renewable energy.

The current study was completed with the development of a model-based application that is able to make predictions based on various input data. In order to validate the 
model, verifiable data from renewable energy databases were analysed. The application was able to assess through stimulation the general behaviour and performance of the system that is modelled based on the input decisions.

\section{Work Methodology}

The model was implemented using the MATLAB program.

The input data represented in matrix correspond to the nine economic indicators (GDP, GDP annual increase rate a PIB, Employment rate, Work productivity, Exports, Total greenhouse gas emissions, Energy intensity, Weight of renewable energy resources out of the total primary energy, Performance of waste recycling) between 2000 and 2013.

The input parameters and the matrix correspond to the nine economic indicators between 2000 and 2014.

Output data is based on the statistical data of the eco-efficiency indicator for a period of 12 months, between 2000 and 2014.

Output data (matrix) is based on the eco-efficiency indicator for a period of 12 months, between 2000 and 2015.

A high-performance algorithm- Levenberg-Marquardt was used for this neuronal network. This algorithm was designed to reach a second-order level training speed. For small and medium-size networks, it is usually applied the LevenbergMarquardt algorithm for training, if enough memory is available. This algorithm can achieve a lower mean square error that any other algorithm.

However, as the number of weights in networks increases, its advantage decreases. This algorithm seems to be the most efficient training method of medium-sized feed-forward neuronal networks, with a moderate size (up to several hundred weights).

An outcome with no poor performance was considered in network designing. The type of network used for developing the prediction model is Feed-forward Backpropagation, trained with Levenberg-Marquardt algorithm, the outcome showed great accuracy for both cases, namely 2014 and 2015.

The input data contain macroeconomic indicators (data) representing the level of economy, according to the statistics provided for each country that was taken into account, between January 2000-December 2013 and January 2000 - December 2014.

The selection of the first study case was based on the idea that if is used the economic indicators from databases regarded as official between 2000 - 2013, a neuronal network can be developed and trained that can subsequently be assessed with data for 2014. The results were synthesized in relevant graphs.

Regarding the second case, the period between 2000 and 2014 was the basis for the neuronal network development and training, keeping the same indicators as in the first case and assessed with output indicator values for 2014. This set of successive tests for the creation, developing, training-learning, testing of a neuronal network was based on the assessment of outcome accuracy provided by this network for various case studies. Neuronal network training, validation and testing with data of economic indicators from the period between 2000 and 2014 
$\diamond \quad$ The input data are made up of 972 parameters, for the period between 2000 and 2013, noted in the model with A matrix.

$\diamond \quad$ The output data are supplied by the values of output indicator for the period between 2000 and 2013, noted in the model with B matrix, containing 108 values.

$\diamond \quad$ Defining the testing matrix - the matrix noted Testin is made up of the nine indicators for the year following the testing, namely 2014, contains 108 values. During the training stage, the following structure of the neurons can be observed:

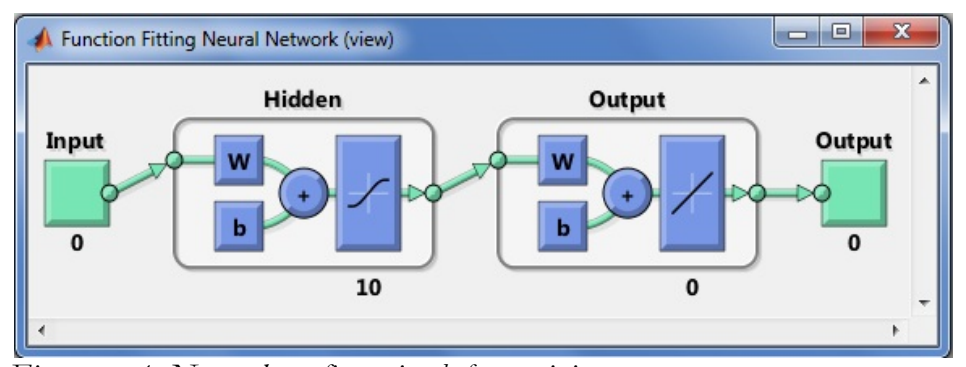

Figure no. 1. Network configuration before training

$\diamond \quad$ Defining the matrix that contains data supplied by official organisms, considered as real data. The Testoutreal matrix is given by the output indicator for the year subsequent to training.

$\diamond \quad$ The network can be assessed by calling the SIM function, having as net parameters the network previously trained with the values plus another parameter representing the matrix of testing values. The resulting values are stored in a Testoutnoted matrix

$\diamond \quad$ The model performance, noted as perfTest, was calculated using the mean square error indicator. 


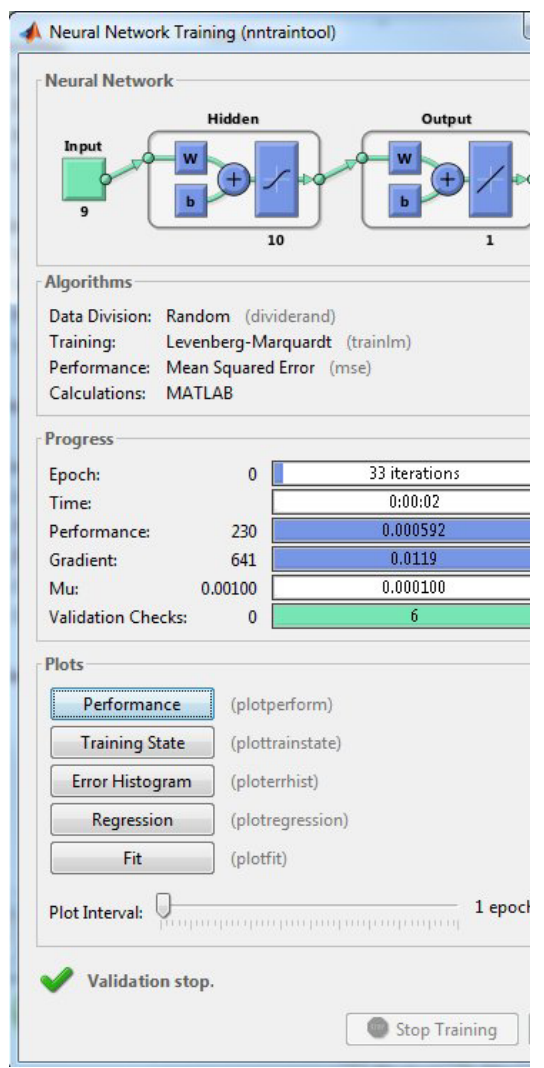

Figure no. 2. Training a network with experimental data

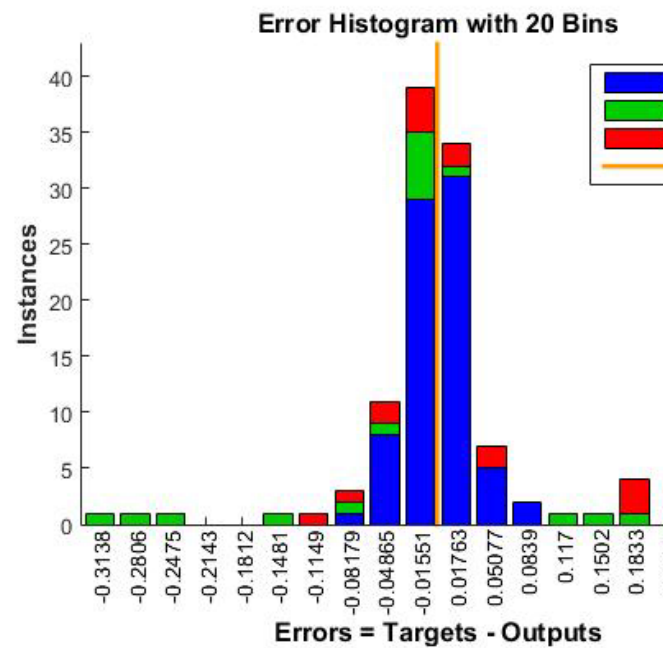

Figure no. 3. Error Histogram

$\diamond \quad$ The number of epochs or the number of training epochs, acquisition and testing is noted as numEpochs. The maximum number of testing epochs is set as 1000 . $\diamond \quad$ The linear regression noted as $\mathrm{R}$, called correlation coefficient, shows great accuracy of the model, between the real data and the simulated ones. 

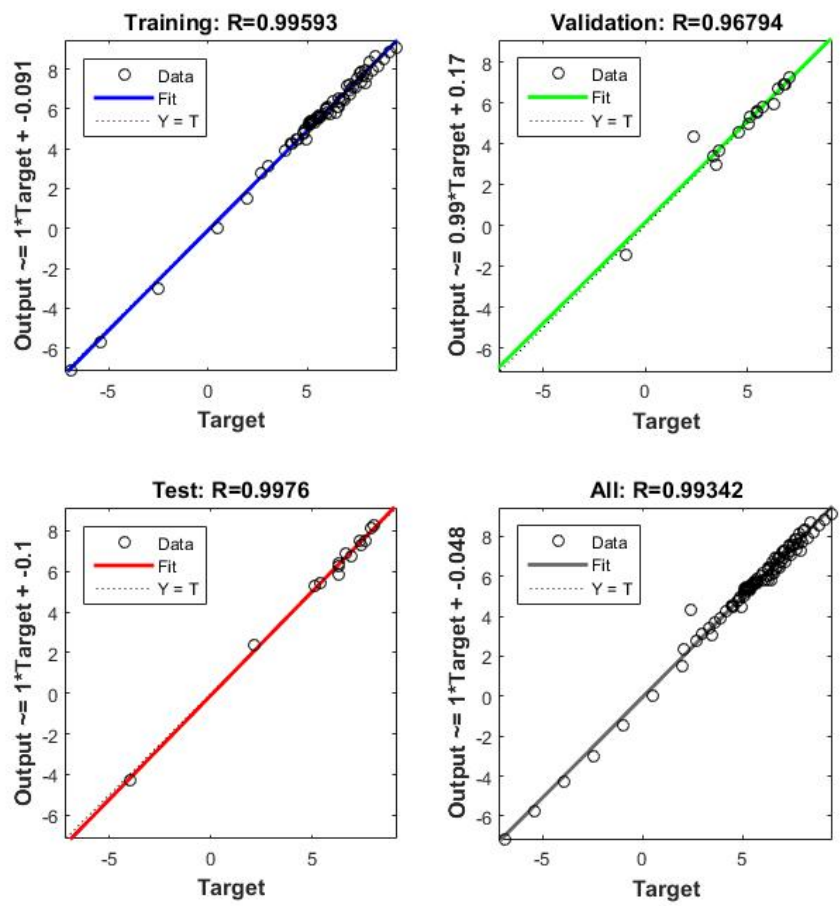

Figure no. 4. Training and validation of neuronal network

The regression value presented in figure above is proving a very good performance of the model for the period of study of 2013-2014. The correlation coefficient has values over 0.99 , which show a good correlation of the data for the training and validation and testing.

The recordings that were taken over represent a real database regarding the economic indicators for the specified period of time. By using these data it was developed a model, using the MATLAB program, for predicting the renewable energy impact upon the economy.

\subsection{Motivation of selecting input and output indicators}

The selection of the macroeconomic indicators was based on economic studies, in the attempt to identify which are the most important ones (statistical data) economically and financially, when the assessment of an economic situation is aimed at. The indicators that were taken into account, regarded as relevant for assessing the impact and competitiveness of renewable resources sector upon the economy - input data - are the following:

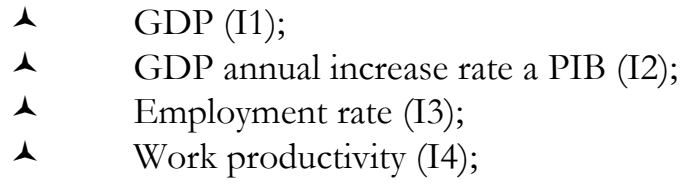




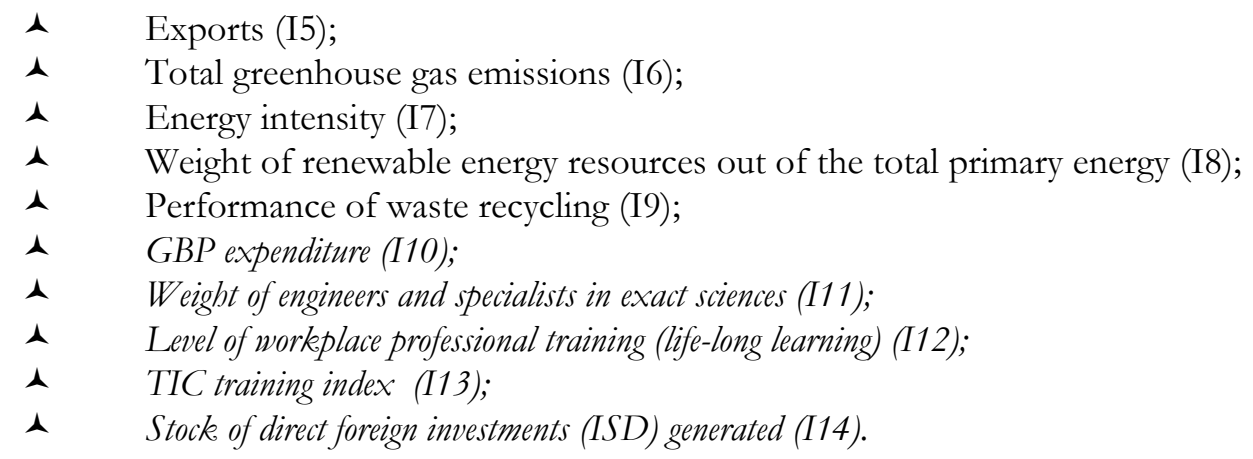

The above-mentioned economic indicators were selected taking into account the criterion of their importance in any economy overview.

The output regarding the economic efficiency is given by the eco-efficiency indicator. Applying the eco-efficiency practices and technologies contributes to the productivity increase, improving environmental performance, reducing costs based on a more efficient use of energy and raw materials resources and the risk of designing new products with less-polluting substances. Due to eco-efficiency, an increase in income occurs.

Also due to the application of eco-efficiency principles helps create new opportunities in terms of research, development, innovation, awareness and empowerment of energy suppliers and consumers, sustain reengineering process, marketing and sales.

\subsection{Building the prediction model}

In order to build a prediction model, certain economic indicators were identified in the initial stage, indicators that have a great impact upon the economy in general. In this regard, the economic indicators considered important in economic forecast were compared, analysed and modelled in the present study by using the artificial intelligence. Choosing the neuronal systems for the prediction model was based on the fact that this type of system is very reliable and runs with minimum maintenance.

The prediction model was built and tested using the neuronal networks with 9 input parameters, in matrix form, 10 neurons in the hidden layer and an output matrix with one parameter as it is shown in the following figure. 


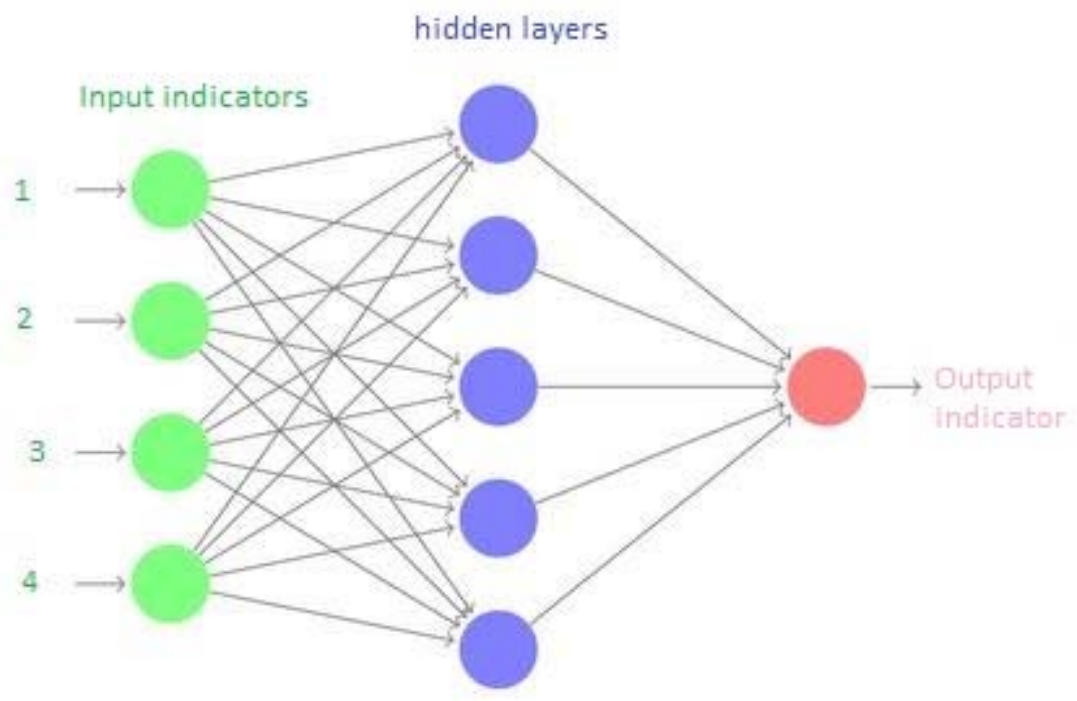

Figure no. 5. The architecture of neural network - input/output indicators

These parameters were analysed for the period 2000 and 2013, in two stages, in order to check the provisioned data, this endeavour helped optimizing the prediction model. In order to achieve a good performance with a predictive network, a priority was its training with representative data for the real economic process. Training an artificial neuronal network based on indicators that cover the entire operating range, provides the best representation and generalization.

In the first stage were used in modelling data specific to period 2000-2013, testing modelling performance with data from 2014. This type of analysis was chosen to observe the variations of economic indicators starting with 2014. During this stage, ER data set were trained, simulated and tested using artificial intelligence. Also the eco-efficiency indicator evolution was monitored, noticing and analysing important aspects for 2013 and 2014.

In the second stage were used officially-declared economic data (internationally) of the economic indicators considered important in economic forecasting for the period 20002014.

The economic results for the national economy are the output of aggregate economic agents, validated by the market and knowledge and analysis of their evolution is very important in practice. Thus, these economic results represent the starting point for decision-making regarding future orientation of attracting and using the factors of production, setting dimensions, structure, quality, supply and demand of economic goods, etc.

The modern research starts from statistical hypotheses on the observed phenomenon, the logical consequences that are deduced being then compared with the available data. If they are consistent, the hypotheses are justified, at least until new more accurate observations. One of the main objectives of statistics is the assessment of uncertainty of inductive conclusions. 


\section{Results and discussion}

In MATLAB program, in the tests that were conducted, the output data sets are made up of statistical data, supplied internationally by official statistical studies, of Romania's eco-efficiency indicator.

In order to identify this process, a neuronal network was designed with the same input vector $\boldsymbol{u}$ and must generate a $\boldsymbol{y}_{\boldsymbol{m}}$ vector that estimates $\boldsymbol{y}_{\boldsymbol{p}}$ to minimize the indicator error.

$\mathrm{e}=\mathrm{y}_{\mathrm{p}}-\mathrm{y}_{\mathrm{m}}$

The most important characteristic of neuronal networks is, in this case, their capacity to model both linear and nonlinear systems.

The designing of this model started from the necessity of prevision intervals to assess the uncertainty of social and economic predictions. The neuronal network thus built and trained can be used both for diagnosis and prediction through stimulation.

The training of the neuronal network was achieved by executing a relatively small number of cycles, at the same time being ensured the convergence of the training process, when the training outputs had been statistically determined.

The structure of the neuronal network is described by the number of neurons and levels of network, determining the modelling tool.

The provisioned intervals were calculated and monitored, based on the mean absolute error (MAE) and on mean squared error (MSE), indicators used by bodies accredited at the national and European level for forecasting.

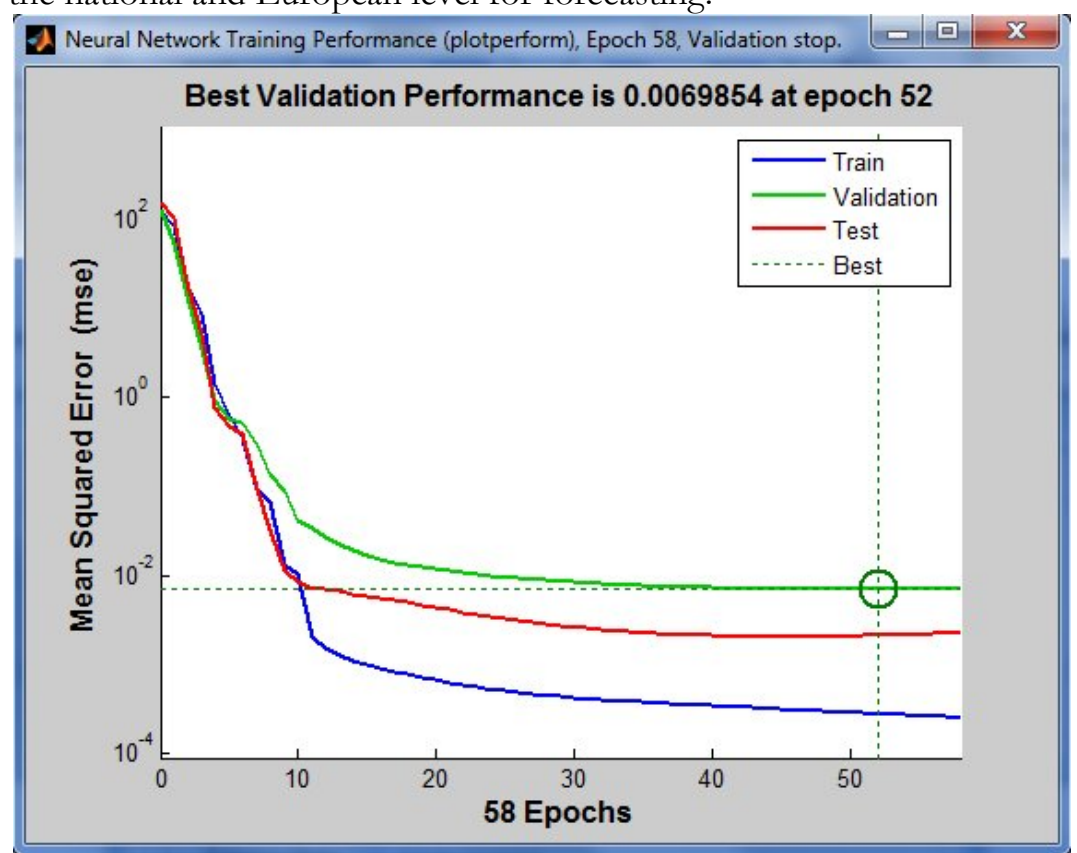

Figure no. 6. The performance of the neural network 
The number of the epochs, or the number of training cycles, learning and testing is designated numEpochs. The maximum number of epochs of the test is set at 1000 .

numEpochs $=$ tr.num_epochs

numEpochs $=52$.

In the model, the data were partitioned in several training, validation and testing sets. Then, the training algorithm is executed on the training set until MSE begins to decrease on the validation set - which appears before reaching the minimum MSE on the training set.

The role of forecast error indicators is to quantify the forecasting errors thus becoming the selection criteria in different methods.

We consider the forecast error indicators at time $\boldsymbol{t}$ as being the difference between the forecasted value for the time $\boldsymbol{t}$ and the actual value registered in time $\boldsymbol{t}$. [Ghinea, M.; Fireteanu, V.; 2001]

$\mathrm{e}_{\mathrm{t}}=\mathrm{Y}_{\mathrm{t}}-\mathrm{P}_{\mathrm{t}}$

where:

$e_{\mathrm{t}}$ - forecasting error at $\boldsymbol{t}$ time,

$\mathrm{Y}_{\mathrm{t}}$ - value forecasted for $\boldsymbol{t}$ time,

$\mathrm{P}_{\mathrm{t}}-$ actual value registered for $\boldsymbol{t}$ time.

If approached statistically, the most common performance function is the mean squared error (MSE) of the error signal represented by the difference between the expected signal and the output.

In general as indicators for forecasting errors are used the mean squared error (MSE) defined as a risk function that corresponds to the expected value of the squared error loss, i.e. it assesses the mean of squared error. By analogy for calculating MSE, we can consider the estimator as the forecasted value of growth rate (rc_p), and the parameter as its actual value (rc_e) and will result the following:

$\operatorname{MSE}\left(\mathrm{rc} \_\mathrm{p}\right)=\operatorname{Var}\left(\mathrm{rc} \_\mathrm{p}\right)+\left[\operatorname{Bias}\left(\mathrm{rc} \_\mathrm{p}, \mathrm{rc} \_\mathrm{e}\right)\right]^{2}$

The higher difference between the value of MAE and that of MSE, the greater the variability of the data sets. 


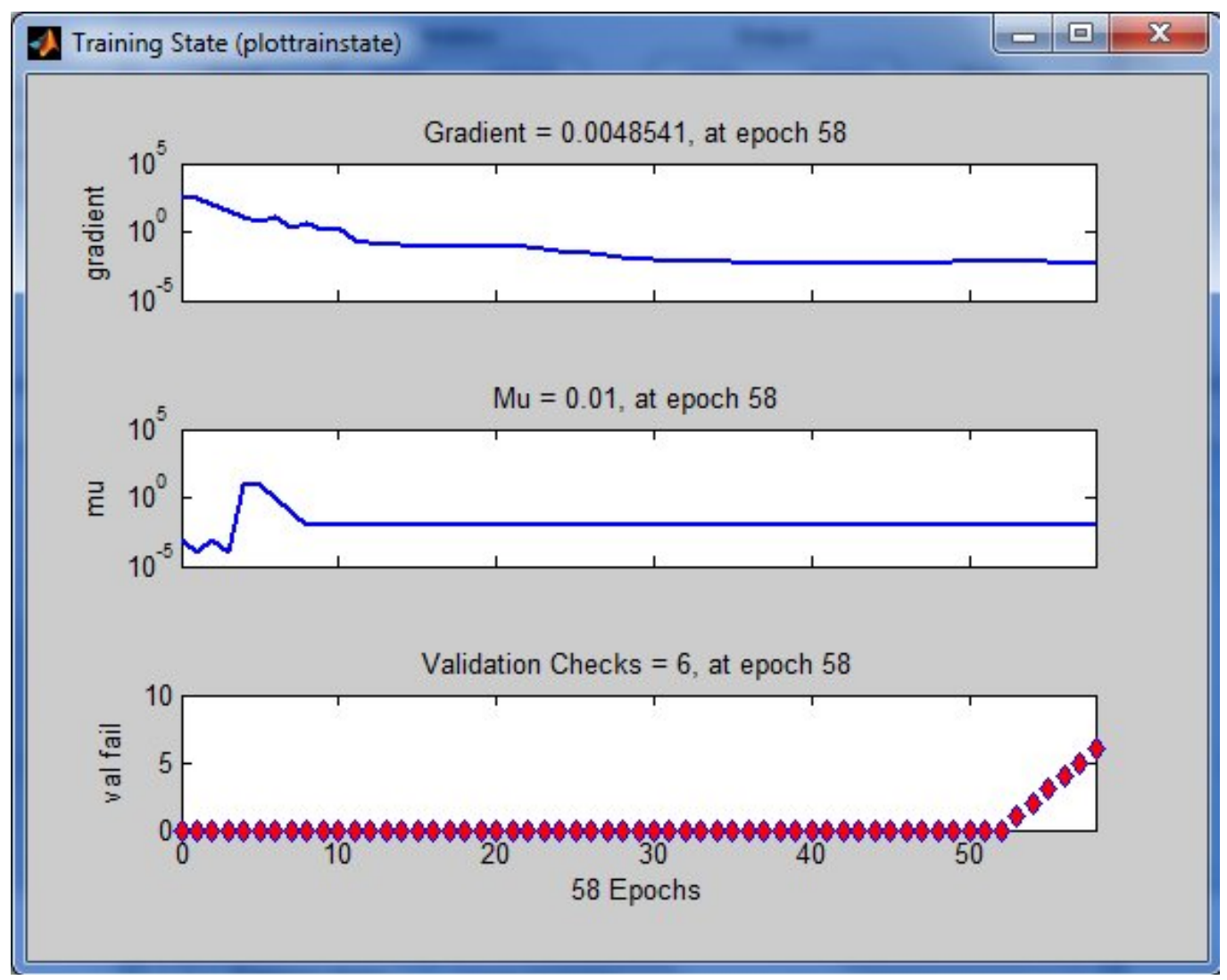

Figure no. 7. Validation of network performance

The gradient value reflects how the weights have changed till have stabilized, in this case stabilized at epoch 58. The difference between the actual and the forecasted value using neural network model is smaller, and indicates that the model provides predictability with a greater degree of accuracy. 


\section{The real value vs the forecasted value}

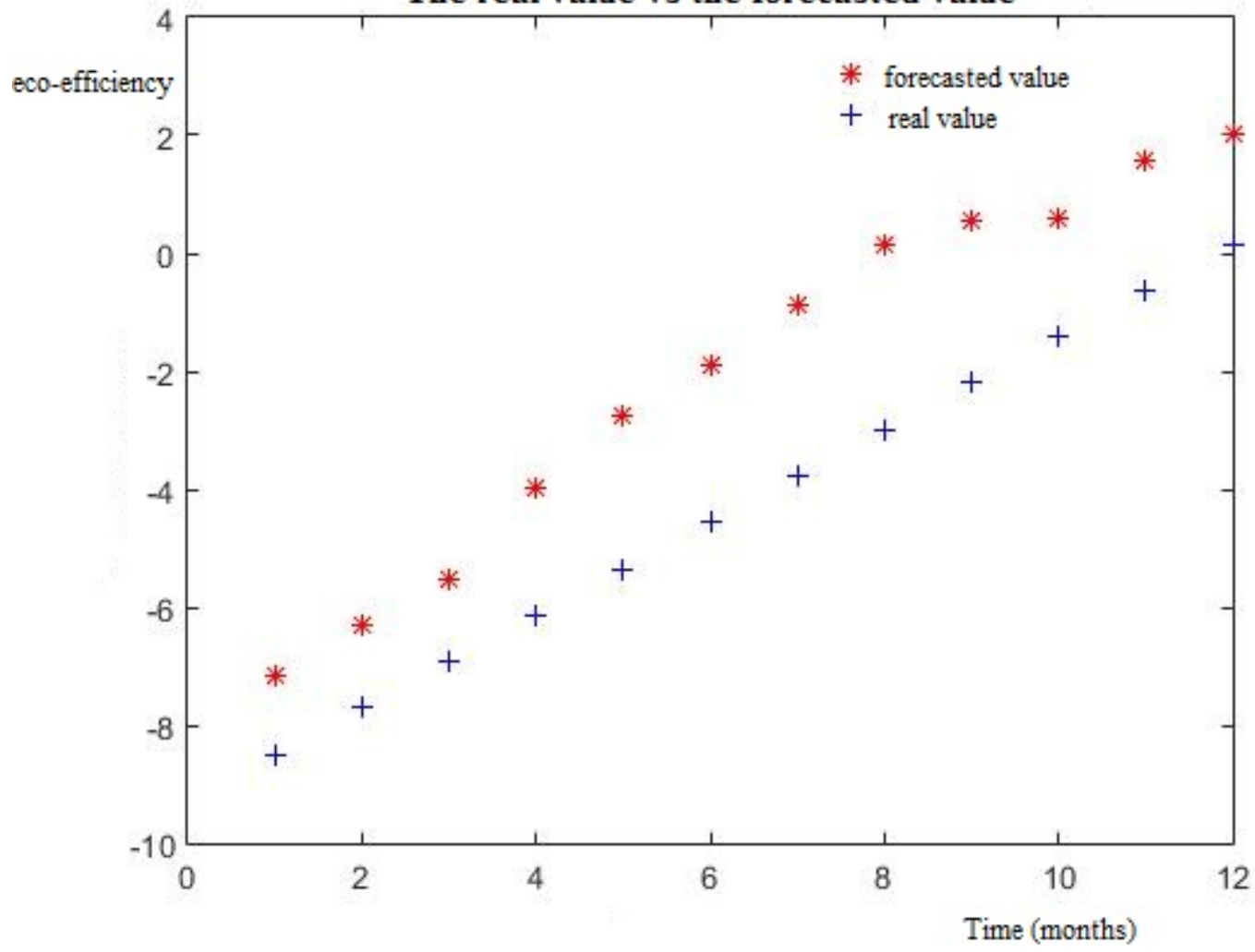

Figure no. 8. The real value vs the forecasted value for România

Following the previous figure can be noticed a slight delay to the actual values from those projected in the year 2014 of the indicator of eco-efficiency indicator. The biggest difference between the actual and forecasted value using neural network model is $\sim 3 \%$ at the level of December. This is considered to be very small difference, resulting developed that the model provides a very good predictability. It is observed that the biggest difference between the actual and forecasted value by using neural network model is $\sim 12 \%$ to the level of March as it was considered to be an acceptable difference, resulting developed that the model provides a satisfactory predictability.

In order to achieve the forecasting characteristic, the influence of past values of the modelled indicator was considered upon its present values. For this reason, the model shows the prediction particularity of the current values of the output variable by adjusting a function depending on the input and output past values. At the time $t$, the output is determined by $\boldsymbol{x}$ input at different past moments $\left(\mathrm{t}\right.$-input $\mathrm{t}_{1}, \ldots, \mathrm{t}_{\text {-input }}$ ) and $\boldsymbol{y}$ output at past times (t-output $1, \ldots$, t-output $\left._{n}\right)$.

In the analyses we conducted, it can be noticed that the results we obtained have similar values, therefore we can conclude that the selection of the nine economic factors influencing renewable energy could enable us to predict accurately this resource, when 
the external factors that have a great impact upon the social and economic environment remain the same.

\section{Conclusions}

The present research aimed at developing and training an artificial neuronal network that was tested and validated with input-output sets, obtained by economic and statistical methods. The current study presented significant contributions able to provide theoretical aspects in developing some projects that can be applied in economy, economic forecasting models, by using artificial intelligence techniques, models posing a challenge to the IT system as well.

A major conclusion that can be drawn is that by using simultaneously classical forecasting methods with the ones used in artificial intelligence, new models can be developed, able to supply more accurate and better structured data than each of these methods taken separately.

Starting from these premises, a prediction model for economic growth was designed and tested using neuronal networks with 9 input parameters, in the matrix form, 10 neurons in the hidden layer and an output matrix with one parameter. The high performance algorithm Levenberg-Marquardt was used to build this neuronal network that operates on sets and designed to reach a second-order level training speed. It can be observed that the technique we used has led to satisfactory output results.

The main concerned was how to build the structure of the neuronal network, the number of layers and neurons corresponding to each of them, so that the results to be as close to truth as possible and the objective of the experiment to be achieved. Thus there were calculated and monitored the differences between the values resulted due to the prediction model achieved by using neuronal networks and the official values supplied by bodies accredited internationally for renewable energy. In order to verify the accuracy of the model, the output data were compared with the actual data, officially provided by authorities in economic field.

For further research, we can identify potential for improvement by data reorganizing in time and space as well as changing the utilities input-output indicator. An attempt can also be made in developing the existing model so that it can automatically retrieve the data supplied by the authorities and in implementing a monitoring function to generate reports at predefined regular intervals.

\section{Acknowledgements:}

The research was supported by Project SOP HRD/159/1.5/S/138963 - PERFORM.

\section{References}

Alam, S., Kaushik, S.C. and Garg, S.N. 2006. Computation of beam solar radiation at normal incidence using artificial neural network, Renewable Energy, Vol. 31, pp.1483-1491.

Calvert, K., \& Simandan, D. (2010). Energy, space, and society: a reassessment of the changing landscape of energy production, distribution, and use. Journal of Economics and Business Research, 16(1), 1337. 
Cao, J. C., \& Cao, S. H. (2006). Study of forecasting solar irradiance using neural networks with preprocessing sample data by wavelet analysis. Energy, 31(15), 3435-3445.

Çetin, A., \& Vardar, A. (2014). The Development of Renewable Energy Sources in Turkey. European Journal of Sustainable Development, 3(4), 365-376

Feng, X., Li, Q., Zhu, Y., Hou, J., Jin, L., \& Wang, J. (2015). Artificial neural networks forecasting of PM 2.5 pollution using air mass trajectory based geographic model and wavelet transformation. Atmospheric Environment, 107, 118-128.

Geman, S., C.H. Hwang.(1982). Nonparametric Maximum Likelihood Estimation by the Method of Sieves, Annals oj Statistics 70, 401-414.

Ghinea, M. ,V. Firețeanu, (2001). MATLAB. Calcul numeric. Grafică. Aplicații, Editura Teora, Bucureşti, pp. 112.

Global National Security and Intelligence Agencies Handbook Volume 1 Strategic Information and Important Contacts, Int'l Business Publications, USA, ISBN 0-7397-9140-0, 2015

Grenander, U. (1981). Abstract Inference. New York: Wiley.

Hymans, S. H., (2008). Forecasting and Econometric Models, The Concise Encyclopedia of Economics.. Library of Economics and Liberty.

Kalogirou, S.A. (2001). Artificial neural networks in renewable energy systems applications: a review', Renewable and Sustainable Energy Reviews,.373-401.

Khaligh, A., \& Onar, O. C. (2009). Energy harvesting: solar, wind, and ocean energy conversion systems. CRC press.

Kornienko, A.A., Kornienko, A.V., Fofanov, O.B., Chubik, M. P. (2015). Knowledge in Artificial Intelligence Systems: Searching the Strategies for ApplicationOriginal Research Article Procedia Social and Behavioral Sciences, 166, 589-594.

Mellit A. (2008). Artificial Intelligence technique for modelling and forecasting of solar radiation data: a review, Journal International Journal of Artificial Intelligence and Soft Computing archive, Volume 1 Issue 1, November 2008, 52-76.

Parker, D., (1982). Learning logic. Invention report 581-64, Department of Electrical Engineering, Stanford University, Stanford., 581(64). Available at: http://dl.acm.org/citation.cfm?id=1212847.

Rumelhart, D. E., G. E. Hinton, and R.J.W., (1986). Learning representations by backpropagation errors. Nature, 323(9), 533-536.

Rumelhart, D.E., Hinton, G.E. \& Williams, R.J., (2013). Learning Internal Representations by Error Propagation. Readings in Cognitive Science: A Perspective from Psychology and Artificial Intelligence, 399-421.

Russell, Stuart; Norvig Peter (2003). Artificial Intelligence: A Modern Approach, 2003.

Simon, H. A. (1955). A behavioral model of rational choice. The quarterly journal of economics, 99-118.

Sims, R. E., Schock, R. N., Adegbululgbe, A., Fenhann, J. V., Konstantinaviciute, I., Moomaw, W., \& Uchiyama, Y. (2007). Energy supply. In Climate change 2007: Mitigation. Contribution of Working Group III to the fourth assessment report of the Intergovernmental Panel on Climate Change. Cambridge University Press.

Sözen, A., Arcaklıoğlu, E., Özalp, M., \& Çağlar, N. (2005). Forecasting based on neural network approach of solar potential in Turkey. Renewable Energy, 30(7), 1075-1090.

Speirs, J., McGlade, C., \& Slade, R. (2015). Uncertainty in the availability of natural resources: Fossil fuels, critical metals and biomass. Energy Policy, 87, 654-664.

White, H., (1988). Economic prediction using neural networks: The case of IBM daily stock returns. Neural Networks, IEEE International Conference on, pp.451 - 458. Available at: http://ieeexplore.ieee.org/xpls/abs_all.jsp?arnumber $=23959$.

Ya, A. Z. (2016). French Green Growth Paradigm In-line with EU Targets Towards Sustainable Development Goals. European Journal of Sustainable Development, 5(2), 143-170.

Yáñez-Arancibia A., Dávalos-Sotelo R., Day J. W. (2014). Ecological Dimensions for Sustainable Socio Economic Development, WIT Press.

Zhang X.;Qi, T.; Karplus, V. (2013). The Energy and CO2 Emissions Impact of Renewable Energy Development in China, Joint Program on the Science and Policy of Global Change, Cambridge, Report No. 242. 